01

\title{
Антиклассическое приближение в задаче об отражении электромагнитной волны от неоднородной среды
}

\author{
(C) B.B. Шагаев \\ Московский государственный технический университет им. Н.Э. Баумана, Калужский филиал, \\ 248000 Калуга, Россия \\ e-mail: shagaev_vv@rambler.ru
}

Поступило в Редакцию 13 апреля 2021 г.

В окончательной редакции 29 августа 2021 г.

Принято к публикации 1 сентября 2021 г.

Выведены выражения для коэффициентов отражения электромагнитных волн с поляризациями $p$ - и $s$-типа от полубесконечной диэлектрической среды, имеющей неоднородный слой. Влияние слоя было учтено методом теории возмущений в квадратичном по толщине слоя приближении. Предложен способ преобразования выражений, выведенных с помощью теории возмущений, в другие, дающие более точные значения. Проведено сравнение угловых зависимостей коэффициента отражения, рассчитанных по выведенным формулам, с зависимостями, полученными методом численного решения уравнений электродинамики. Сформулированы требования к характеристикам слоя, позволяющие минимизировать погрешность аналитического решения.

Ключевые слова: диэлектрическая среда, неоднородный слой, коэффициент отражения электромагнитной волны, аналитический расчет, теория возмущений.

DOI: 10.21883/JTF.2022.01.51845.106-21

\section{Введение}

Интерес к процессам отражения и пропускания электромагнитных волн диэлектрическими материалами стимулируется развитием таких направлений, как оптоэлектроника, радиотехнические системы, оптика полупроводников и полимеров. Также внимание многих исследователей привлекают метаматериалы - искусственно синтезированные среды, обладающие нехарактерными для естественных сред электромагнитными свойствами. Неоднородные диэлектрические слои могут использоваться как составная часть метаматериала.

Задача об отражении электромагнитной волны от плоской границы среды, характеризуемой неоднородным профилем диэлектрической проницаемости, не имеет общего аналитического решения. Волновое уравнение, являющееся основой расчета коэффициента отражения, может быть преобразовано в уравнение Гельмгольца, и для неоднородной среды точное решение уравнения известно только в частных случаях. Классическим методом нахождения точного решения является метод замены переменных и функций, позволяющий преобразовать исходное уравнение в какое-либо стандартное. Данный метод был использован для нахождения решений в случаях линейного, параболического и экспоненциального профиля диэлектрической проницаемости. К настоящему времени разработаны методы редукции уравнений электродинамики, в которых замена переменных дополнена преобразованием компонент волнового поля с помощью вспомогательных функций [1-3]. Условия таких преобразований определяют широкие классы непрерывных распределений профиля диэлектрической проницаемости, допускающих точные аналитические представления для компонент электромагнитного поля. Для среды с произвольной координатной зависимостью материальных параметров используют либо численные, либо приближенные аналитические методы [4-10]. При медленном изменении показателя преломления на расстоянии длины волны эффективен метод квазиклассического приближения, также известный как метод ВКБ (Вентцеля-Крамерса-Бриллюэна). Противоположным случаем является среда, в которой параметры существенно меняются на расстоянии длины волны (антиклассическое приближение). В частности, к настоящему времени теоретически и экспериментально исследовано отражение электромагнитной волны от неоднородной структуры, состоящей из слоев с субволновыми толщинами $[11,12]$. Оказалось, что модель, основанная на замене такой структуры эффективной средой с однородными свойствами, не дает правильных характеристик отражения.

В настоящей работе рассмотрена еще одна задача антиклассического приближения - задача об отражении электромагнитной волны от среды с неоднородным слоем субволновой толщины. Полагалось, что отражающие свойства среды характеризуется диэлектрической проницаемостью и что область неоднородности имеет малую фазовую толщину. Последнее требование позволило применить методы теории возмущений и получить приближенные аналитические выражения для коэффициента отражения. По аналогии с работами [1-3] компоненты волнового поля были выражены через вспомогательные функции, и был разработан метод расчета этих функций. Кроме того, была предложена модель, основанная на замене неоднородного слоя однородным с эффективными параметрами. Такая замена расширила возможности 
аналитического представления коэффициента отражения при переходе к более толстым слоям. Математический аппарат разработанной теории представляет интерес для анализа волновых полей и в других областях физики сплошных сред.

\section{1. Математическая модель}

Геометрические аспекты модели изображены на рис. 1. Неоднородный слой моделируется координатной зависимостью диэлектрической проницаемости $\varepsilon(z)$. Геометрию слоя определяют два параметра, которые характеризуют глубину залегания и толщину $-\Delta$ и $\delta$ соответственно. Полагалось, что по краям слоя функция $\varepsilon(z)$ приближается к асимптотическому значению $\varepsilon(+\infty)$ не медленнее, чем экспонента. Такое требование обеспечит сходимость интегралов, входящих в итоговые формулы и содержащих функцию $\varepsilon(z)$.

В задаче об отражении электромагнитной волны важную роль играет поляризация волны. В настоящей работе рассмотрены волны с линейными поляризациями $p$ - и $s$-типов. Подробные расчеты приведены для волн с $p$-поляризацией, а для волн с $s$-поляризацией приведены лишь окончательные выражения.

В используемой системе координат (рис. 1) вектор напряженности магнитного поля волны с $p$-поляризацией

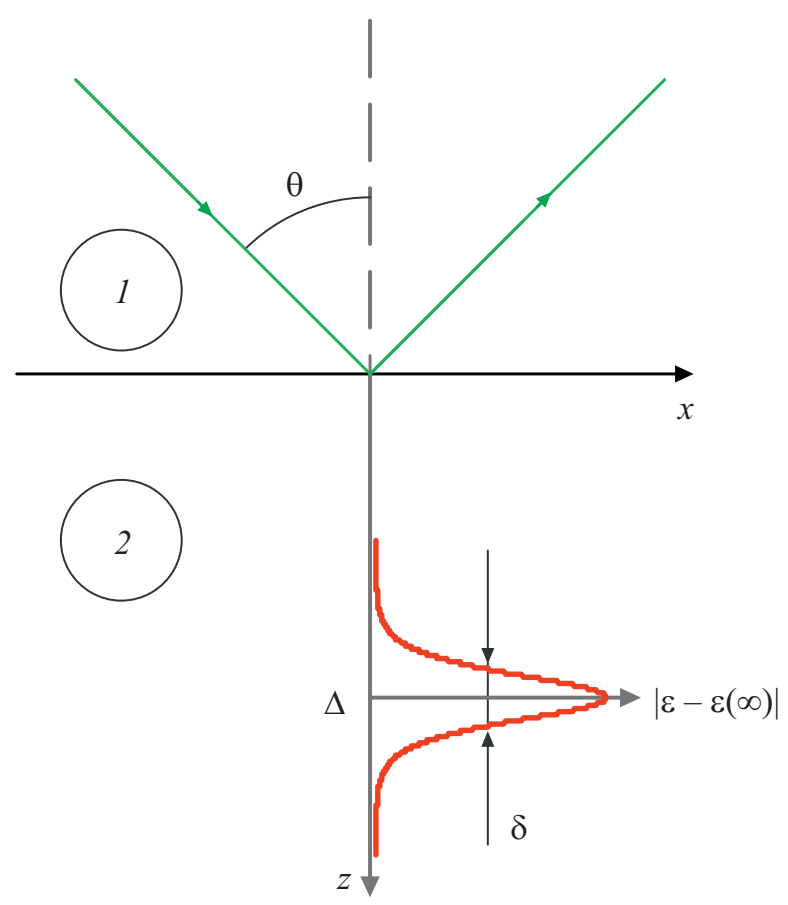

Рис. 1. Геометрические параметры модели отражения электромагнитной волны от среды с неоднородным слоем. Координатные оси $x$ и $z$ расположены в плоскости падения луча. Граница раздела между вакуумом 1 и отражающей средой 2 совпадает с плоскостью $z=0$. Условно изображено отклонение диэлектрической проницаемости в неоднородном слое от асимптотического значения. Геометрия слоя характеризуется расстоянием до границы раздела $\Delta$ и толщиной $\delta$. можно представить в виде

$$
\mathbf{H}=\left[\begin{array}{c}
0 \\
h(z) \\
0
\end{array}\right] \cdot \exp i\left(\omega t-k_{x} x\right),
$$

где $\omega-$ круговая частота волны, $t-$ время, $k_{x}=\omega \sin \theta / c$ и $c-$ скорость света в вакууме, $i-$ мнимая единица. Из уравнений электродинамики следует дифференциальное уравнение, определяющее функцию $h(z)$ (в гауссовой системе единиц измерения)

$$
\frac{1}{\varepsilon(z)} \frac{d}{d z}\left[\frac{1}{\varepsilon(z)} \frac{d h(z)}{d z}\right]+k_{p}^{2}(z) h(z)=0 .
$$

Функция $k_{p}(z)$ определена формулой (индексом „р“ отмечен тип поляризации)

$$
k_{p}^{2}(z)=\frac{1}{\varepsilon^{2}(z)}\left[\frac{\omega^{2}}{c^{2}} \varepsilon(z)-k_{x}^{2}\right] .
$$

Координатную ось $z$ разделим на три области и зададим вид функции $h(z)$ в каждой из этих областей с помощью выражений:

$$
h(z)=\left\{\begin{array}{cc}
a_{1} \exp \left(-i k_{z} z\right)+b_{1} \exp \left(i k_{z} z\right), & z<0, \\
a_{2}(z) \exp \left[-i k_{p} \varepsilon(\xi) d \xi\right] & \\
+b_{2}(z) \exp \left[i k_{p} \int_{0}^{z} \varepsilon(\zeta) d \zeta\right], & 0<z<\Delta, \\
a_{3}(z) \exp \left[-i k_{p} \int_{\Delta}^{z} \varepsilon(\xi) d \zeta\right] & \\
+b_{3}(z) \exp \left[i k_{p} \varepsilon(\zeta) d \xi\right], & \Delta<z,
\end{array}\right.
$$

где $k_{z}=\omega \cos \theta / c$ и введено обозначение: $k_{p}=k_{p}(+\infty)$.

Коэффициент отражения волны от среды выражается формулой

$$
R_{p}=\left|\frac{b_{1}}{a_{1}}\right|^{2} .
$$

Преобразуем формулу, используя условие непрерывности тангенциальных компонент напряженностей магнитного и электрического поля волны (компоненты $H_{y}$ и $E_{x}$ соответственно) на границе $z=0$. Учитывая, что уравнения электродинамики позволяют выразить $E_{x}$ через $H_{y}$, можно использовать условие непрерывности для отношения компонент

$$
\frac{H_{y}}{E_{x}}=-\frac{i \omega}{c} \frac{h(z)}{[1 / \varepsilon(z)][d h(z) / d z]} .
$$

Подставляя вместо $h(z)$ выражения из (3), получим следующее соотношение:

$$
\frac{b_{1}}{a_{1}}=\frac{r_{p}+V_{p}}{1+r_{p} V_{p}} .
$$


Здесь введены обозначения $V_{p}=b_{2}(0) / a_{2}(0)$ и

$$
r_{p}=\frac{k_{z}-k_{p}}{k_{z}+k_{p}}
$$

Таким образом, задача по расчету коэффициента отражения может быть сведена к задаче по расчету значения параметра $V_{p}$ и подстановке этого значения в формулу

$$
R_{p}=\left|\frac{r_{p}+V_{p}}{1+r_{p} V_{p}}\right|^{2} .
$$

Поскольку в интервале $0<z<\Delta$ вместо одной функции $h(z)$ введены две $\left(a_{2}(z)\right.$ и $\left.b_{2}(z)\right)$, на эти функции можно наложить дополнительное условие. Выберем следующее условие:

$$
\begin{aligned}
& \frac{d a_{2}(z)}{d z} \exp \left[-i k_{p} \int_{0}^{z} \varepsilon(\varsigma) d \varsigma\right] \\
& +\frac{d b_{2}(z)}{d z} \exp \left[i k_{p} \int_{0}^{z} \varepsilon(\varsigma) d \varsigma\right]=0 .
\end{aligned}
$$

Тогда из (1) следует уравнение

$$
\begin{aligned}
& \frac{i k_{p}}{\varepsilon(z)} \frac{d a_{2}(z)}{d z} \exp \left[-i k_{p} \int_{0}^{z} \varepsilon(\varsigma) d \varsigma\right]-\frac{i k_{p}}{\varepsilon(z)} \frac{d b_{2}(z)}{d z} \\
& \times \exp \left[i k_{p} \int_{0}^{z} \varepsilon(\varsigma) d \varsigma\right]=\left[k_{p}^{2}(z)-k_{p}^{2}\right] h(z) .
\end{aligned}
$$

Уравнения (7) и (8) с помощью алгебраических преобразований можно привести к виду

$$
\frac{d a_{2}(z)}{d z}=\frac{\varepsilon(z)}{2 i k_{p}}\left[k_{p}^{2}(z)-k_{p}^{2}\right] h(z) \exp \left[i k_{p} \int_{0}^{z} \varepsilon(\varsigma) d \varsigma\right],
$$

$\frac{d b_{2}(z)}{d z}=-\frac{\varepsilon(z)}{2 i k_{p}}\left[k_{p}^{2}(z)-k_{p}^{2}\right] h(z) \exp \left[-i k_{p} \int_{0}^{z} \varepsilon(\varsigma) d \varsigma\right]$.

Функция $h(z)$ в этих уравнениях задана формулой (3) в интервале $0<z<\Delta$.

Аналогичными действиями можно получить уравнения, связывающие между собой функции $a_{3}(z), b_{3}(z)$ и $h(z)$ в области $\Delta<z$ :

$$
\begin{gathered}
\frac{d a_{3}(z)}{d z}=\frac{\varepsilon(z)}{2 i k_{p}}\left[k_{p}^{2}(z)-k_{p}^{2}\right] h(z) \exp \left[i k_{p} \int_{\Delta}^{z} \varepsilon(\varsigma) d \varsigma\right], \\
\frac{d b_{3}(z)}{d z}=-\frac{\varepsilon(z)}{2 i k_{p}}\left[k_{p}^{2}(z)-k_{p}^{2}\right] h(z) \exp \left[-i k_{p} \int_{\Delta}^{z} \varepsilon(\varsigma) d \varsigma\right] .
\end{gathered}
$$

Уравнения (9)-(12) будут использованы для нахождения отношения $b(0) / a_{2}(0)$, необходимого для расчета коэффициента отражения.

\section{2. Теория возмущений}

Преобразуем уравнения (9)-(12) в интегральную форму, подставив выражения для функции $h(z)$ из (3) в каждой из областей:

$$
\begin{aligned}
& a_{2}(z)-a_{2}(0)=\frac{1}{2 i k_{p}} \int_{0}^{z} \varepsilon(\varsigma)\left[k_{p}^{2}(\varsigma)-k_{p}^{2}\right]\left\{a_{2}(\varsigma)\right. \\
& \left.+b_{2}(\varsigma) \exp \left[2 i k_{p} \int_{0}^{\Delta} \varepsilon(\eta) d \eta\right] \exp \left[2 i k_{p} \int_{\Delta}^{\varsigma} \varepsilon(\eta) d \eta\right]\right\} d \varsigma, \\
& b_{2}(z)-b_{2}(0)=-\frac{1}{2 i k_{p}} \int_{0}^{z} \varepsilon(\varsigma)\left[k_{p}^{2}(\varsigma)-k_{p}^{2}\right]\left\{a_{2}(\varsigma)\right. \\
& \left.\times \exp \left[-2 i k_{p} \int_{0}^{\Delta} \varepsilon(\eta) d \eta\right] \exp \left[-2 i k_{p} \int_{\Delta}^{\varsigma} \varepsilon(\eta) d \eta\right]+b_{2}(\varsigma)\right\} d \varsigma, \\
& a_{3}(+\infty)-a_{3}(z)=\frac{1}{2 i k_{p}} \int_{z}^{+\infty} \varepsilon(\varsigma)\left[k_{p}^{2}(\varsigma)-k_{p}^{2}\right] \\
& \times\left\{a_{3}(\varsigma)+b_{3}(\varsigma) \exp \left[2 i k_{p} \int_{\Delta}^{\varsigma} \varepsilon(\eta) d \eta\right]\right\} d \varsigma, \\
& b_{3}(+\infty)-b_{3}(z)=-\frac{1}{2 i k_{p}} \int_{z}^{+\infty} \varepsilon(\varsigma)\left[k_{p}^{2}(\varsigma)-k_{p}^{2}\right] \\
& \times\left\{a_{3}(\varsigma) \exp \left[-2 i k_{p} \int_{\Delta}^{\varsigma} \varepsilon(\eta) d \eta\right]+b_{3}(\varsigma)\right\} d \varsigma .
\end{aligned}
$$

Отсутствие отраженной волны при $z \rightarrow+\infty$ дает $b_{3}(+\infty)=0$.

Для построения теории возмущений в задаче должен быть малый параметр. Наложим на толщину слоя $\delta$ следующее ограничение (условие малой фазовой толщины слоя):

$$
\left|k_{p} \max \{\varepsilon(z)\}\right| \delta \ll 1 .
$$

Нулевое приближение теории возмущений соответствует однородной полубесконечной среде с $\varepsilon(z)=\varepsilon(+\infty)$ для всех $z>0$. В этом приближении $a_{2}(z)=a_{3}(z)=a_{2}(0), \quad b_{2}(z)=b_{3}(z)=b_{2}(0)=0$. При выполнении условия (17) правые части уравнений (13)-(16) можно рассматривать как возмущения, определяющие координатную зависимость функций $a_{2}(z), a_{3}(z), b_{2}(z), b_{3}(z)$. В первом приближении 
данные функции можно получить, подставив в правые части уравнений константы нулевого приближения. Также в этом приближении можно полагать, что $\exp \left[ \pm 2 i k_{p} \int_{\Delta}^{5} \varepsilon(\eta) d \eta\right] \approx 1$. Тогда уравнения $(13)-(16)$ примут вид

$$
\begin{gathered}
a_{2}(z)-a_{2}(0)=I_{2}(z) \frac{a_{2}(0)}{2 i k_{p}} \\
b_{2}(z)-b_{2}(0)=-I_{2}(z) \frac{a_{2}(0)}{2 i k_{p}} \exp \left[-2 i k_{p} \int_{0}^{\Delta} \varepsilon(\eta) d \eta\right] \\
a_{3}(+\infty)-a_{3}(z)=I_{3}(z) \frac{a_{2}(0)}{2 i k} \\
b_{3}=I_{3}(z) \frac{a_{2}(0)}{2 i k_{p}}
\end{gathered}
$$

Здесь введены функции, значения которых имеют первый порядок малости по толщине неоднородного слоя $(\sim \delta)$ :

$$
\begin{aligned}
I_{2}(z) & =\int_{0}^{z} \varepsilon(\varsigma)\left[k_{p}^{2}(\varsigma)-k_{p}^{2}\right] d \varsigma, \\
I_{3}(z) & =\int_{z}^{+\infty} \varepsilon(\varsigma)\left[k_{p}^{2}(\varsigma)-k_{p}^{2}\right] d \varsigma .
\end{aligned}
$$

Поправки второго приближения можно получить, подставив в правые части уравнений $(13)-(16)$ координатные зависимости первого приближения. Кроме того, в этом приближении нужно использовать уточняющие замены $\exp \left[ \pm 2 i k_{p} \int_{\Delta}^{5} \varepsilon(\eta) d \eta\right] \approx 1 \pm 2 i k_{p} \int_{\Delta}^{5} \varepsilon(\eta) d \eta$, и в конечных выражениях нужно отбросить слагаемые третьего порядка малости $\left(\sim \delta^{3}\right)$. Тогда во втором приближении получим равенства

$$
\begin{aligned}
& a_{2}(\Delta)=a_{2}(0)+\frac{I_{2}(\Delta)}{2 i k_{p}} \\
& \times\left\{a_{2}(0)+b_{2}(0) \exp \left[2 i k_{p} \int_{0}^{\Delta} \varepsilon(\eta) d \eta\right]\right\} \\
& +G_{2}(\Delta) b_{2}(0) \exp \left[2 i k_{p} \int_{0}^{\Delta} \varepsilon(\eta) d \eta\right], \\
& b_{2}(\Delta)=b_{2}(0)-\frac{I_{2}(\Delta)}{2 i k_{p}}{ }^{\Delta}\left[\begin{array}{c}
\Delta \\
\left.a_{2}(0) \exp [\eta) d \eta\right]+b_{2}(0)
\end{array}\right\} \\
& +G_{0}(\Delta) a_{2}(0) \exp \left[\begin{array}{l}
-2 i k_{p} \\
\int_{0} \varepsilon(\eta) d \eta
\end{array}\right]
\end{aligned}
$$

$$
\begin{gathered}
a_{3}(\Delta)=a_{3}(+\infty)-\frac{I_{3}(\Delta)}{2 i k_{p}} a_{3}(+\infty), \\
b_{3}(\Delta)=\frac{I_{3}(\Delta)}{2 i k_{p}} a_{3}(+\infty)-G_{3}(\Delta) a_{3}(+\infty) .
\end{gathered}
$$

Здесь $G_{2}(\Delta)$ и $G_{3}(\Delta)$ - значения функций:

$$
\begin{aligned}
G_{2}(z) & =\int_{0}^{z} \varepsilon(\varsigma)\left[k_{p}^{2}(\varsigma)-k_{p}^{2}\right]\left[\int_{\Delta}^{\varsigma} \varepsilon(\eta) d \eta\right] d \varsigma, \\
G_{3}(z) & =\int_{z}^{+\infty} \varepsilon(\varsigma)\left[k_{p}^{2}(\varsigma)-k_{p}^{2}\right]\left[\int_{\Delta}^{\varsigma} \varepsilon(\eta) d \eta\right] d \varsigma .
\end{aligned}
$$

Использование условия непрерывности отношения (4) при $z=\Delta$ приводит к уравнению

$$
\begin{gathered}
\frac{a_{2}(\Delta) \exp \left[-i k_{p} \int_{0}^{\Delta} \varepsilon(\eta) d \eta\right]+b_{2}(\Delta) \exp \left[k_{p} \int_{0}^{\Delta} \varepsilon(\eta) d \eta\right]}{-k_{p} a_{2}(\Delta) \exp \left[-i k_{p} \int_{0}^{\Delta} \varepsilon(\eta) d \eta\right]+k_{p} b_{2}(\Delta) \exp \left[k_{p} \int_{0}^{\Delta} \varepsilon(\eta) d \eta\right]} \\
=\frac{a_{3}(\Delta)+g_{3}(\Delta)}{-k_{p} a_{3}(\Delta)+k_{p} b_{3}(\Delta)} .
\end{gathered}
$$

Подставив сюда выражения (18)-(21) и выполнив элементарные преобразования, получим уравнение для нахождения отношения $V_{p}=b_{2}(0) / a_{2}(0)$ :

$$
\begin{gathered}
1+G_{2}(\Delta)+\left[1+G_{2}(\Delta)\right] V_{p} \exp \left[2 i k_{p} \int_{0}^{\Delta} \varepsilon(\eta) d \eta\right] \\
\hline-k_{p}+i I_{2}(\Delta)+k_{p} G_{2}(\Delta)+\left[k_{p}+i I_{2}(\Delta)-k_{p} G_{2}(\Delta)\right] \times \\
\quad \times V_{p} \exp \left[2 i k_{p} \int_{0}^{\Delta} \varepsilon(\eta) d \eta\right] \\
=\frac{G_{3}(\Delta)-1}{k_{p}+i I_{3}(\Delta)+k_{p} G_{3}(\Delta)} .
\end{gathered}
$$

Уравнение является алгебраическим, и его решение во втором порядке теории возмущений можно преобразовать к виду

$$
V_{p}=-\left(\frac{i I}{2 k_{p}}+\frac{I^{2}}{4 k_{p}^{2}}+G\right) \exp \left[-2 i k_{p} \int_{0}^{\Delta} \varepsilon(\eta) d \eta\right],
$$

где введены обозначения

$$
\begin{gathered}
I=I_{2}(\Delta)+I_{3}(\Delta)=\int_{0}^{+\infty} \varepsilon(\varsigma)\left[k_{p}^{2}(\varsigma)-k_{p}^{2}\right] d \varsigma \\
G=G_{2}(\Delta)+G_{3}(\Delta)=\int_{0}^{+\infty} \varepsilon(\varsigma)\left[k_{p}^{2}(\varsigma)-k_{p}^{2}\right]\left[\int_{\Delta}^{\varsigma} \varepsilon(\eta) d \eta\right] d \zeta .
\end{gathered}
$$


Параметр $I$ линейно зависит от толщины слоя $(I \sim \delta)$, а параметр $G$ - квадратично $\left(G \sim \delta^{2}\right)$.

Существенным недостатком формулы (22) является то, что для выполнения расчета требуется как функция $\varepsilon(z)$, так и отдельно параметр $\Delta-$ он входит в показатель экспоненты и в выражение параметра $G$. Преобразуем формулу (22) к такому виду, в котором параметр $\Delta$ отсутствует. Оставаясь в рамках второго приближения, формулу (22) можно представить в виде

$$
\begin{aligned}
& V_{p} \approx-\frac{i I}{2 k_{p}} \\
& \times \exp \left\{-i\left[2 k_{p} \int_{0}^{\Delta} \varepsilon(\eta) d \eta+\frac{I}{2 k_{p}}+2 k_{p} \frac{G}{I}\right]\right\} .
\end{aligned}
$$

Введем функцию

$$
I(z)=\int_{z}^{+\infty} \varepsilon(\varsigma)\left[k_{p}^{2}(\varsigma)-k_{p}^{2}\right] d \varsigma .
$$

Параметр $G$ можно выразить через эту функцию

$$
G=-\int_{0}^{+\infty} \frac{d I(\varsigma)}{d \varsigma}\left[\int_{\Delta}^{\varsigma} \varepsilon(\eta) d \eta\right] d \varsigma
$$

Интегрируя по частям и учитывая равенства $I(0)=I$ и $I(+\infty)=0$, получим равенство

$$
\begin{aligned}
& 2 k_{p} \int_{0}^{\Delta} \varepsilon(\eta) d \eta+\frac{I}{2 k_{p}}+2 k_{p} \frac{G}{I} \\
& =\frac{1}{2 k_{p} I}\left[I^{2}+4 k_{p}^{2} \int_{0}^{+\infty} \varepsilon(\varsigma) I(\varsigma) d \varsigma\right] .
\end{aligned}
$$

Для следующего этапа преобразования введем функцию

$$
Y(z)=I^{2}(z)+4 k_{p}^{2} \int_{z}^{+\infty} \varepsilon(\varsigma) I(\varsigma) d \varsigma .
$$

Дифференцируя функцию и выполняя необходимые подстановки, можно получить уравнение

$$
\frac{d Y(z)}{d z}=-2 \varepsilon(z)\left[k_{p}^{2}(z)+k_{p}^{2}\right] I(z) .
$$

Интегрируя это уравнение и учитывая, что $Y(+\infty)=0, \quad$ а $Y(0)$ совпадает с выражением, заключенным в скобки в правой части формулы (24), получим

$$
\begin{aligned}
& I^{2}+4 k_{p}^{2} \int_{0}^{+\infty} \varepsilon(\varsigma) I(\varsigma) d \varsigma \\
& =2 \int_{0}^{+\infty} \varepsilon(z)\left[k_{p}^{2}(z)+k_{p}^{2}\right]\left\{\int_{z}^{+\infty} \varepsilon(\varsigma)\left[k_{p}^{2}(\varsigma)-k_{p}^{2}\right] d \varsigma\right\} d z .
\end{aligned}
$$

Выполняя подстановку данного выражения в равенство (24) и далее в (23), получим вместо (22) соотношение

$$
V_{p}=-\frac{i}{2 k_{p}} \int_{0}^{+\infty} \varepsilon(z)\left[k_{p}^{2}(z)-k_{p}^{2}\right] d z \cdot \exp \left(-2 i k_{p} \Lambda_{p}\right)
$$

где

$$
\begin{aligned}
& \Lambda_{p}= \\
& \frac{\int_{0}^{+\infty} \varepsilon(z)\left[k_{p}^{2}(z)+k_{p}^{2}\right]\left\{\int_{z}^{+\infty} \varepsilon(\varsigma)\left[k_{p}^{2}(\varsigma)-k_{p}^{2}\right] d \varsigma\right\} d z}{2 k_{p}^{2} \int_{0}^{+\infty} \varepsilon(z)\left[k_{p}^{2}(z)-k_{p}^{2}\right] d z} .
\end{aligned}
$$

В итоге коэффициент отражения может быть рассчитан по формуле (6) с подстановками выражений (25), (26). Данный расчет основан только на зависимости и не содержит в явном виде параметров $\Delta$ и $\delta$.

\section{3. Усовершенствование формул теории возмущений}

Существуют модели с точным аналитическим решением задачи об отражении электромагнитной волны. Одна из них - модель с однородным слоем. В разд. 3 описан способ экстраполяции формул, полученных для такой модели, на модель с неоднородным слоем. Способ позволяет преобразовать выведенные формулы теории возмущений в более точные.

Коэффициент отражения волны с $p$-поляризацией от среды с однородным слоем может быть рассчитан с помощью выражения

$$
R_{p}=\left|\frac{r_{p}+V_{p u}}{1+r_{p} V_{p u}}\right|^{2}
$$

Параметр $r_{p}$ задан соотношением (5), а параметр $V_{p u}-$ следующим точным выражением:

$$
V_{p u}=\frac{1-\exp \left(-i \varphi_{p u}\right)}{1-r_{p u}^{2} \exp \left(-i \varphi_{p u}\right)} r_{p u} \exp \left(-i \Phi_{p u}\right),
$$

где

$$
\begin{gathered}
r_{p u}=\frac{k_{p}-k_{p u}}{k_{p}+k_{p u}}, \quad k_{p u}^{2}=\frac{1}{\varepsilon_{u}^{2}}\left[\frac{\omega^{2}}{c^{2}} \varepsilon_{u}-k_{x}^{2}\right], \\
\varphi_{p u}=2 k_{p u} \varepsilon_{u} \delta, \quad \Phi_{p u}=2 k_{p} \varepsilon_{d} \Delta_{u} .
\end{gathered}
$$

Здесь $\varepsilon_{u}$ - диэлектрическая проницаемость однородного слоя толщиной $\delta$, расположенного в среде с диэлектрической проницаемостью $\varepsilon_{d}$ между плоскостями $z=\Delta_{u}$ и $z=\Delta_{u}+\delta$. 
Для слоя с малой фазовой толщиной $\left(\left|\varphi_{p u} \ll 1\right|\right)$ во втором порядке теории возмущений из выражения (28) получим

$$
V_{p u} \approx i \varphi_{p u} \frac{k_{p}^{2}-k_{p u}^{2}}{4 k_{p} k_{p u}} \exp \left[-i\left(\Phi_{p u}+\frac{k_{p}^{2}+k_{p u}^{2}}{4 k_{p} k_{p u}} \varphi_{p u}\right)\right] .
$$

Формулу (29) можно преобразовать к виду (25), заменив параметры $k_{p u}, \varphi_{p u}, \Phi_{p u}$ подходящими выражениями. Приравнивая правые части формул (25) и (29), которые являются комплексными величинами, можно составить два уравнения для получения искомых замен. Однако двух уравнений будет недостаточно, чтобы получить выражения для замены трех параметров. Поэтому нужно использовать дополнительное условие, налагаемое на предполагаемые замены. В настоящей работе в качестве такого условия была выбрана замена $k_{p u} \rightarrow k_{p l}$, в которой параметр $k_{p l}$ задан следующим выражением:

$$
k_{p l}^{2}=\frac{\int_{0}^{+\infty} k_{p}^{2}(z)\left[k_{p}^{2}(z)-k_{p}^{2}\right] \varepsilon(z) d z}{\int_{0}^{+\infty}\left[k_{p}^{2}(z)-k_{p}^{2}\right] \varepsilon(z) d z} .
$$

Следует отметить, что параметр $k_{p l}$ можно интерпретировать как среднее значение функции $k_{p}(z)$ в неоднородном слое. В частности, применительно к однородному слою данная формула приводит к равенству $k_{p l}=k_{p u}$. Иными словами, предложенное выражение соответствует физическому смыслу заменяемого параметра.

Равенство множителей перед экспонентами в формулах (25), (29) с учетом замены $k_{p u} \rightarrow k_{p l}$ позволяет вывести выражение для замены $\varphi_{p u} \rightarrow \varphi_{p u}$ :

$$
\varphi_{p}=-\frac{2 k_{p l}}{k_{p}^{2}-k_{p l}^{2}} \int_{0}^{+\infty} \varepsilon(z)\left[k_{p}^{2}(z)-k_{p}^{2}\right] d z .
$$

Равенство показателей экспонент с учетом уже сделанных замен приводит к еще одной замене $\Phi_{p u} \rightarrow \Phi_{p}$ :

$$
\Phi_{p}=2 k_{p} \Lambda_{p}-\frac{k_{p}^{2}+k_{p l}^{2}}{4 k_{p} k_{p l}} \varphi_{p}
$$

По аналогии с переходом от формулы теории возмущений (29) к точной формуле (28), преобразуем формулу (25) к виду

$$
\begin{gathered}
V_{p l}=\frac{1-\exp \left(-i \varphi_{p}\right)}{1-r_{p l}^{2} \exp \left(-i \varphi_{p}\right)} r_{p l} \exp \left(-i \Phi_{p}\right), \\
r_{p l}=\frac{k_{p}-k_{p l}}{k_{p}+k_{p l}}
\end{gathered}
$$

Итоговое выражение коэффициента отражения имеет вид

$$
R_{p}=\left|\frac{r_{p}+V_{p l}}{1+r_{p} V_{p l}}\right|^{2}
$$

Данная формула имеет ту же структуру, что и формула (6), но с параметром $V_{p l}$ вместо $V_{p}$.

В заключение раздела приведем краткие сведения об усовершенствовании выражения для коэффициента отражении волны с $s$-поляризацией. При проведении расчетов для этого типа волны был использован тот же подход, что и для волны с $p$-поляризацией.

Волна с $s$-поляризацией имеет вектор напряженности электрического поля следующего вида (рис. 1):

$$
\mathbf{E}=\left[\begin{array}{c}
0 \\
e(z) \\
0
\end{array}\right] \cdot \exp i\left(\omega t-k_{x} x\right)
$$

Волновое уравнение, составляющее основу расчетов, имеет вид

$$
\frac{d^{2} e(z)}{d z^{2}}+k_{s}^{2}(z) e(z)=0 .
$$

Функция $k_{s}^{2}(z)$ определена формулой

$$
k_{s}^{2}(z)=\frac{\omega^{2}}{c^{2}} \varepsilon(z)-k_{x}^{2} .
$$

Условию непрерывности подчиняются компоненты $E_{y}$ и $H_{x}$. Их отношение можно рассчитать по формуле

$$
\frac{E_{y}}{H_{x}}=\frac{i \omega}{c} \frac{e(z)}{[d e(z) / d z]} .
$$

Расчет коэффициента отражения по теории возмущений приводит к следующим соотношениям:

$$
\begin{gathered}
R_{s}=\left|\frac{r_{s}+V_{s}}{1+r_{s} V_{s}}\right|^{2}, \\
V_{s}=-\frac{i}{2 k_{s}} \int_{0}^{+\infty}\left[k_{s}^{2}(z)-k_{s}^{2}\right] d z \cdot \exp \left(-2 i k_{s} \Lambda_{s}\right), \\
\Lambda_{s}=\frac{\int_{0}^{+\infty}\left[k_{s}^{2}(z)+k_{s}^{2}\right]\left\{\int_{z}^{+\infty}\left[k_{s}^{2}(\varsigma)-k_{s}^{2}\right] d \varsigma\right\} d z}{2 k_{s}^{2} \int_{0}^{+\infty}\left[k_{s}^{2}(z)-k_{s}^{2}\right] d z}, \\
r_{s}=\frac{k_{z}-k_{s}}{k_{z}+k_{s}}, \\
k_{z}=\omega \cos \theta / c \quad \text { и } k_{s}=k_{s}(+\infty) .
\end{gathered}
$$

Усовершенствованная формула для коэффициента отражения имеет вид

$$
R_{s}=\left|\frac{r_{s}+V_{s l}}{1+r_{s} V_{s l}}\right|^{2},
$$

где

$$
V_{s l}=\frac{1-\exp \left(-i \varpi_{s}\right)}{1-r_{s l}^{2} \exp \left(-i \varphi_{s}\right)} r_{s l} \exp \left(-i \Phi_{s}\right)
$$




$$
\begin{gathered}
\varphi_{s}=-\frac{2 k_{s l}}{k_{s}^{2}-k_{s l}^{2}} \int_{0}^{+\infty}\left[k_{s}^{2}(z)-k_{s}^{2}\right] d z, \\
k_{s l}^{2}=\frac{\int_{0}^{+\infty} k_{s}^{2}(z)\left[k_{s}^{2}(z)-k_{s}^{2}\right] d z}{\int_{0}^{+\infty}\left[k_{s}^{2}(z)-k_{s}^{2}\right] d z}, \\
r_{s l}=\frac{k_{s}-k_{s l}}{k_{s}+k_{s l}}, \\
\Phi_{s}=2 k_{s} \Lambda_{s}-\frac{k_{s}^{2}+k_{s l}^{2}}{4 k_{s} k_{s l}} \varphi_{s} .
\end{gathered}
$$

Формулы (35) и (41) с подстановками выражений входящих в них параметров позволяют рассчитать коэффициент отражения, используя зависимость $\varepsilon(z)$. В частности, для среды с однородным слоем данные формулы дают точные выражения для коэффициента отражения при любой толщине слоя. Напротив, формулы теории возмущений (для $p$-поляризации это формулы (6), (25), (26), а для $s$-поляризации - (37)-(39)) даже в этом простом случае являются всего лишь приближенными.

\section{4. Тестовые расчеты и их обсуждение}

Тестовые расчеты были выполнены для неоднородных сред, в которых координатная зависимость диэлектрической проницаемости описывается формулой Эпштейна [13]:

$$
\varepsilon(z)=\varepsilon_{\infty}+4 \varepsilon_{l} \frac{\exp \left[\left(z-z_{l}\right) / \delta_{l}\right]}{\left\{1+\exp \left[\left(z-z_{l}\right) / \delta_{l}\right]\right\}^{2}},
$$

где $\varepsilon_{\infty}-$ асимптотическое значение диэлектрической проницаемости при $z \rightarrow \infty, \varepsilon_{l}=\varepsilon\left(z_{l}\right), z_{l}-$ глубина распложения слоя, $\delta_{l}$ - параметр, характеризующий толщину слоя.

Для оценки точности аналитического решения было выполнено сравнение угловых зависимостей коэффициента отражения, рассчитанных по выведенным формулам, с угловыми зависимостями, полученными численным методом из уравнений электродинамики.

В численных методах широко применяются различные интерпретации рекуррентного метода и метода матриц переноса. В обоих методах неоднородную среду заменяют структурой с тонкими однородными слоями. В каждом таком слое уравнения электродинамики имеют решения в виде проходящей и отраженной волны. Требование непрерывности тангенциальных составляющих напряженностей электрического и магнитного полей приводят к системе алгебраических уравнений, связывающим между собой амплитуды волн в соседних слоях. Такая система составляет основу расчета коэффициента отражения в обоих методах. В настоящей работе для численного расчета был использован рекуррентный метод. В этом методе каждый слой характеризуется своим амплитудным коэффициентом отражения - отношением амплитуды отраженной волны к амплитуде проходящей. Последовательным переходом от наиболее удаленного слоя к поверхностному можно рассчитать все локальные коэффициенты. Предполагается также, что за внешней границей самого удаленного слоя среда становится практически однородной. С помощью электродинамических граничных условий полный коэффициент отражения может быть выражен через локальный коэффициент примыкающего к поверхности слоя. Уменьшая толщину каждого слоя и увеличивая их общую толщину, можно добиться необходимой точности расчета коэффициента отражения от всей неоднородной структуры.

На рис. 2 приведены примеры расчета. В одном случае диэлектрическая проницаемость внутреннего слоя имела мнимую часть (рис. 2, $a, b)$, в другом - была вещественной со значением в минимуме $\min \{\varepsilon(z)\}=1$ (рис. 2,c,d). Кроме того, был выполнен расчет для системы из двух слоев (рис. 2,e,f).

Из анализа угловых зависимостей коэффициента отражения следует, что точность расчетов по усовершенствованным формулам существенно выше, чем по формулам теории возмущений. Расчеты с использованием разных значений толщины слоя показали, что усовершенствованные формулы хорошо описывают угловые зависимости для значений $\delta_{l} / \lambda \leq 0.02$, а формулы теории возмущений - только для $\delta_{l} / \lambda \leq 0.01$ (где $\lambda=2 \pi c / \omega$ длина электромагнитной волны в вакууме). В интервале $0.01 \leq \delta_{1} / \lambda \leq 0.02$ расчет по формулам теории возмущений может приводить к значительным погрешностям. Для значений $\delta_{l} / \lambda \leq 0.01$ угловые зависимости, рассчитанные по формулам теории возмущений и по усовершенствованным формулам, практически не отличаются от рассчитанных численным методом.

Следует также отметить, что на графиках, изображенных на рис. 2, амплитуда осцилляций коэффициента отражения примерно на порядок превосходит отношение $\delta_{l} / \lambda$. Данное обстоятельство указывает на сильное влияние внутреннего слоя на электродинамические характеристики среды даже в случае слоя с субволновой толщиной. Кроме того, предложенная аналитическая модель продемонстрировала высокую чувствительность к выбору значений свободных параметров слоя.

Особое внимание нужно обратить на неоднородные слои, у которых разница $\varepsilon(z)-\varepsilon(\infty)$ имеет значения разного знака на разных интервалах переменной $z$. В этом случае возможно выполнение равенств $\int_{0}^{+\infty} \varepsilon(z)\left[k_{p}^{2}(z)-k_{p}^{2}\right] d z=0$ или $\int_{0}^{+\infty}\left[k_{s}^{2}(z)-k_{s}^{2}\right] d z=0$. Данные интегралы входят в знаменатели выражений (26), (30), (39), (44), и поэтому их нулевое значение недопустимо. В качестве примера была рассмотрена 

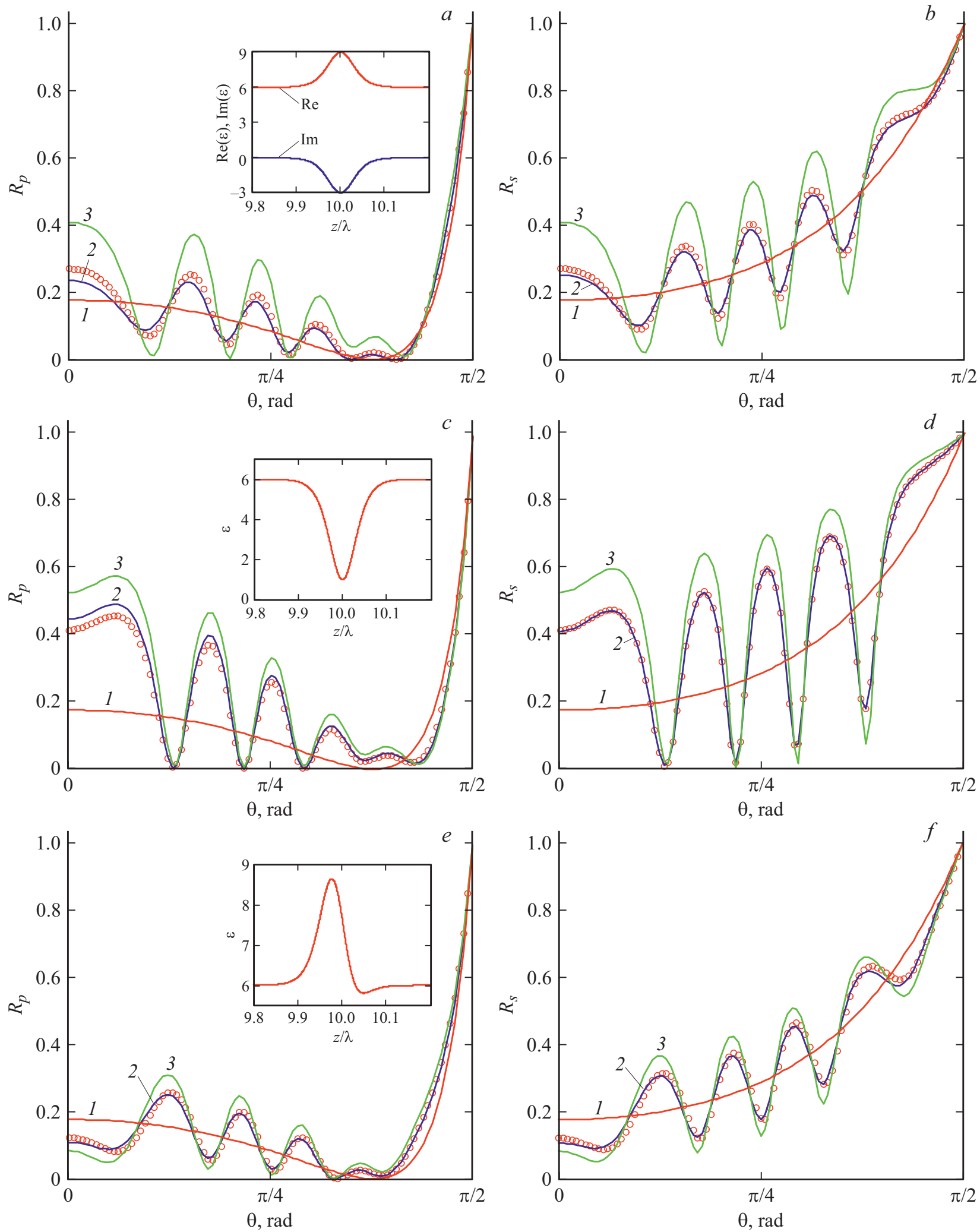

Рис. 2. Угловые зависимости коэффициента отражения для волн с $p$ - и $s$-поляризациями ( $a, c, e$ и $b, d, f$ соответственно). $1-$ для однородной среды, 2 - расчет по усовершенствованным формулам для среды с неоднородным слоем, 3 - расчет по формулам теории возмущений для среды с неоднородным слоем. Кружки - расчет численным методом по уравнениям электродинамики для среды с неоднородным слоем. На вставках изображены координатные зависимости диэлектрической проницаемости $\varepsilon(z)$ в неоднородном слое. Зависимости $\varepsilon(z)$ заданы формулой $(47)(a-d)$ и формулой $(48)(e, f)$. Для всех рассчитанных зависимостей одинаковыми были значения $\varepsilon_{\infty}=6, z_{l}=10 \lambda, \delta_{l}=0.02 \lambda$. Другие значения: $\varepsilon_{l}=3(1-i)(a, b) ; \varepsilon_{l}=-5(c, d) ; \varepsilon_{l}=3, \gamma=0.3(e, f)$. 
среда с двумя слоями Эпштейна (рис. 2,e,f):

$$
\begin{aligned}
& \varepsilon(z)=\varepsilon_{\infty}+4 \varepsilon_{l} \frac{\exp \left\lfloor\left(z-z_{l}+\delta_{l}\right) / \delta_{l}\right\rfloor}{\left\{1+\exp \left[\left(z-z_{l}+\delta_{l}\right) / \delta_{l}\right]\right\}^{2}} \\
& -\gamma \cdot 4 \varepsilon_{l} \frac{\exp \left\lfloor\left(z-z_{l}-\delta_{l}\right) / \delta_{l}\right\rfloor}{\left\{1+\exp \left[\left(z-z_{l}-\delta_{l}\right) / \delta_{l}\right]\right\}^{2}} .
\end{aligned}
$$

В одном слое диэлектрическая проницаемость больше, а в другом меньше, чем $\varepsilon(\infty)$. При выборе значения коэффициента $\gamma=0.3$ указанные интегралы отличны от нуля, и имеется хорошее совпадение аналитических зависимостей с численным решением уравнений электродинамики. При увеличении $\gamma$ погрешность возрастает, а для $\gamma=1$ использование аналитических зависимостей становится вообще недопустимым.

\section{Заключение}

Разработан метод аналитического расчета коэффициента отражения от среды с неоднородным слоем. Метод основан на теории возмущений и на экстраполяции точных формул модели с однородным слоем на неоднородную модель. Предложено характеризовать неоднородный слой параметрами, являющимися обобщением параметров, используемых в модели с однородным слоем. Итогом разработки стали формулы (35), (41) с подстановками, определяемыми аналитическими выражениями. В отличие от численных методов в предложенном методе не требуется послойная дискретизация среды. Все выражения были получены для координатной зависимости диэлектрической проницаемости общего вида $\varepsilon(z)$. Данное обстоятельство существенно упрощает вычислительную сторону задачи и позволяет анализировать многопараметрические модели $\varepsilon(z)$. Тестовые расчеты показали, что использование метода экстраполяции существенно повышает точность расчета коэффициента отражения. Рассчитанные по экстраполяционным формулам угловые зависимости коэффициента отражения достаточно точно воспроизводят интерференционные эффекты отражения. Выведенные формулы могут быть использованы для анализа и прогнозирования свойств материалов с модифицированными слоями. В работе не вводились ограничения на глубину расположения неоднородного слоя, так что полученные результаты можно рассматривать как обобщение результатов, представленных в работе [9]. Кроме того, в той работе существенными были ограничения, связанные с выбором геометрии отражения вблизи угла Брюстера и с отсутствием поглощения в среде. В настоящей работе коэффициент отражения не был ограничен малыми значениями, а диэлектрическая проницаемость могла содержать координатнозависимую мнимую часть.

\section{Конфликт интересов}

Автор заявляет, что у него нет конфликта интересов.

\section{Список литературы}

[1] А.Б. Шварцбург. УФН, 170 (12), 1297 (2000). DOI: 10.3367/UFNr.0170.200012b.1297 [A.B. Shvartsburg. PhysicsUspekhi, 43 (12), 1201 (2000). DOI: 10.1070/PU2000v043n12ABEH000827]

[2] Н.С. Ерохин, Ю.М. Зуева, А.Б. Шварцбург. Квантовая электроника, 43 (9), 785 (2013) [N.S. Erokhin, Yu.M. Zueva, A.B. Shvartsburg. Quant. Electron., 43 (9), 785 (2013). DOI: 10.1070/QE2013v043n09ABEH015209]

[3] А.Б. Шварцбург, М.Б. Агранат, О.В. Чефонов. Квантовая электроника, 39 (10), 948 (2009) [А.В. Shvartsburg, M.B. Agranat, O.V. Chefonov. Quant. Electron., 39 (10), 948 (2009). DOI: 10.1070/QE2009v039n10ABEH014109]

[4] Ю.А. Кравцов, Ю.И. Орлов. Геометрическал оптика неоднородных сред (Наука, М., 1980)

[5] Р.Э. Наурзалин. Журнал радиоэлектроники (электронный журнал), (7) 2007.

URL: http://jre.cplire.ru/win/jul07/4/text.html (дата обращения 01.07.2021)

[6] И.В. Антонец, Л.Н. Котов, В.Г. Шавров, В.И. Щеглов. Журнал радиоэлектроники (электронный журнал), (4) 2013. URL: http://jre.cplire.ru/alt/apr13/6/text.pdf (дата обращения 01.07.2021)

[7] Д.И. Биленко, В.П. Полянская, М.А. Гецьман, Д.А. Горин, А.А. Невешкин, А.М. Ященок. ЖТФ, 75 (6), 69 (2005). [D.I. Bilenko, V.P. Polyanskaya, M.A. Getsman, D.A. Gorin, A.A. Neveshkin, A.M. Yaschenok. Tech. Phys., 50 (6), 742 (2005). DOI: $10.1134 / 1.1947351]$

[8] И.П. Козлов. ЖТФ, 69 (8), 5 (1999) [I.P. Kozlov. Tech. Phys., 44 (8), 873 (1999). DOI: 10.1134/1.1259397]

[9] В.В. Шагаев. ЖТФ, 85 (12), 6 (2015). [V.V. Shagaev. Tech. Phys., 60 (12), 1738 (2015).

DOI: $10.1134 / \mathrm{S} 1063784215120191]$

[10] В.В. Шагаев. Радиотехника и электроника, 63 (2), 126 (2018). DOI: 10.7868/S0033849418020031

[V.V. Shagaev. J. Commun. Technol. Electron., 63 (2), 111 (2018). DOI: 10.1134/S1064226918020109]

[11] H.H. Sheinfux, I. Kaminer, Y. Plotnik, G. Bartal, M. Segev. Phys. Rev. Lett., 113 (24), 243901 (2014).

DOI: 10.1103/PhysRevLett.113.243901

[12] S.V. Zhukovsky, A. Andryieuski, O. Takayama, E. Shkondin, R. Malureanu, F. Jensen, A.V. Lavrinenko. Phys. Rev. Lett., 115 (17), 177402 (2015). DOI: 10.1103/PhysRevLett.115.177402

[13] L.M. Brekhovskikh. Waves in Layered Media, 2nd ed. (Academic Press, NY., 1980) 